\title{
BIOPHOTONICS
}

\section{Selective disinfection}

A photonic scheme for the selective inactivation of viruses shows promise for making blood transfusion safer and helping to treat diseases involving rapidly mutating viral species, such as the human immunodeficiency virus.

By using ultrashort pulsed laser technology, a group of researchers from the USA have demonstrated that they can selectively inactivate a range of both pathogenic and non-pathogenic viruses, leaving surrounding mammalian cells unharmed (J. Biomed. Opt. 14, 064042; 2009).

A drawback of traditional disinfection approaches based on biochemical and pharmaceutical methods is that there is the risk of the target virus becoming drug-resistant. Although illumination with ultraviolet light is a highly effective disinfection method, it has no selectivity, which can damage both viral particles and mammalian cells.

"The main advantage of our approach over existing techniques is that an ultrashort pulsed laser is used to inactivate viruses by mechanical means, and thus offers selectivity," said Kong-Thon Tsen, a researcher from Arizona State University who was involved in the study.

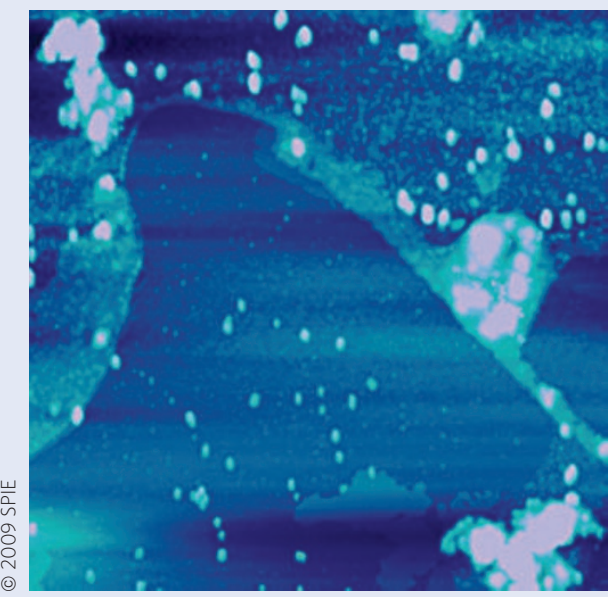

The scheme of Tsen et al. involves $600 \mathrm{fs}, 1.4 \mu \mathrm{J}$ laser pulses from a frequency-doubled 1.55 - $\mu \mathrm{m}$-wavelength fibre laser operated at a repetition rate of $500 \mathrm{kHz}$. The pulses are used to excite large amplitude vibrations in the protein coat of a viral particle - the capsid thus damaging it and inactivating the virus. By proper tuning of the laser power, Tsen and co-workers showed that they could inactivate the desired viruses while leaving the surrounding mammalian cells unharmed.
Three possible mechanisms are considered to be responsible for the vibrations generated in the viral shell: direct excitation of vibrational Ramanactive modes on the capsid; indirect generation of vibrations on the capsid through electronic absorption of the laser; and generation of a shock-wave by the laser. Tsen and co-workers said that more work is required to clarify the key mechanism.

The attraction of the laser scheme is that it is non-invasive and environmentally friendly, requiring no chemicals. Furthermore, the method can inactivate both the wild and mutated strains of viruses. "This intriguing advantage is particularly important for the treatment of diseases that involve rapidly mutating viral species such as AIDS," said Tsen.

In the future it is hoped that this laser method could prove useful for the disinfection of blood components such as platelets, red blood cells and plasmas, making blood transfusion safer. The scheme could also be used in a manner similar to dialysis, making it attractive for the treatment of blood-borne viral diseases in a clinic.

RACHEL WON

\section{QUANTUM OPTICS}

\section{Repairing quadrature entanglement}

Optical loss degrades quantum correlations, hindering the use of quantum quadrature entanglement for many applications. Researchers have now experimentally demonstrated that this entanglement can be recovered using photon subtraction.

\section{Alexei Ourjoumtsev}

$\mathrm{M}$ any experimental techniques, from optical coherence tomography to gravitational-wave detection, require the precise control of coherent light. This precision is often limited by the inevitable quantum fluctuations of amplitude and phase at a level known as the 'shot noise level'. In 1935, Einstein, Podolsky and Rosen realized that these quantum fluctuations, although unavoidable, could be correlated, meaning that two distant beams can in principle have unknown but exactly equal amplitudes and exactly opposite phases ${ }^{1}$. In practice, such 'quadrature-entangled' beams can be produced using nonlinear optics, and their relative fluctuations can be several times smaller than the shot noise level while their individual fluctuations remain large. Apart from being of fundamental interest, quadrature entanglement has many promising applications, mainly in the field of quantum information processing (QIP). There is, however, a major hurdle to overcome: optical losses rapidly degrade quadrature entanglement, and so restoring it has been a long-standing goal.

Now, reporting in Nature Photonics ${ }^{2}$, Takahashi et al. from the University of Tokyo and the National Institute of Communications Technology in Japan describe how they have enhanced the quadrature entanglement between distant beams, succeeding at last to implement a protocol first proposed almost ten years $\mathrm{ago}^{3-5}$. The main idea was to 'distil' a large 